Pub. Mat. UAB

$N^{\circ} 22$ Nov. 1980

Actes VII JMHL

FORMULAS DE CUADRATURA CON GRADO DE PRECISION 2n-l CORRESPONDIENTES A UN NUMERO ARBITRARIO $m$ DE NODOS $Y$ A UNA LOCALIZACION TAMBIEN ARBITRARIA DE LOS MISMOS.

Andrës Arroyo Perez.

Dpto. de Ecuaciones Funcionales

Universidad de Sevilia

Dado un entero positivo $\underline{n}$, existe una formula de cuadratura con precisión $2 n-1$ para la integral en [a,b] de la función $f(x) \in C^{n}[a, b]$, formula definida para un numero variable, m, de nodos y para cualquier localización de los mismos en el intervalo de integración considerado. Se expone como obtener los distintos elementos que definen la fórmula, asi como cotas de error para la misma.

Sí en la expresión general de una formula de cuadratura, (para $f(x) \in c^{n}[a, b], p(x) \subseteq L[a, b]$, siendo $p(x) \neq 0$ en un conjunto de mediala positiva:

$$
\int_{a}^{b} p(x) f(x) d x=\sum_{h=0}^{n-1} \sum_{i=1}^{m} A_{h i} f^{h)}\left(x_{i}\right)+R(F)
$$

con

$$
\begin{gathered}
A_{h i}=\left[L_{n-h-1}^{*}\left(\emptyset_{i}(x)-\emptyset_{i-1}(x)\right)\right]_{x=x_{i}} \\
0 \leq h \leq n-1 ; \quad 1 \leq i \leq m
\end{gathered}
$$


y

$$
R(f)=\sum_{i=0}^{m} \int_{x_{i}}^{x_{i+1}} \phi_{i}(x) L(f(x)) d x
$$

obtenida para una localizacion arbitraria de los nodos:

$$
x_{0}=a \leq x_{1}<x_{2} \cdots \cdots \cdots<x_{m} \leq b=x_{m+1}
$$

$y$ a partir del operador diferencial lineal de orden $n, L$, las funciones $\emptyset_{i}(x)$, soluciones de la ecuación diferencial lineal

$$
L^{*} \varnothing(x)=p(x)
$$

se eligen de forma que cada $\varnothing_{i}(x)$ sea en $\left[x_{i}, x_{i+1}\right], 0 \leq i \leq m$, ortogonal a $L(f(x))$, se tendrá que la fórmula (l) será exacta para toda función $f(x)$ tal que:

$$
\int_{x_{i} \quad x_{i}(x) L(f(x)) d x=0}^{0 \leq i \leq m}
$$

En el trabajo consideramos el caso en que, para cualquier localización de los nodos dados en (4), suponiendo siempre que $x_{1}=a y x_{m}=b$ con lo que no se han de considerar $n i \emptyset_{0}(x)$ ni $\not g_{m}(x)$ en (2) y (3), el operador viene dado por $\mathrm{L}=\mathrm{d}^{\mathrm{n}} / \mathrm{dx^{n }}=\mathrm{D}^{\mathrm{n}}$, $p(x)=1$ y bajo estas hipotesis se obtienen formulas del tipo (1) exactas para todo polinomio de grado menor o igual que $2 n-1$ $y$ esto para cualquier localización del número $m$ de nodos $y$ de su localización.

En este caso, al ser $L^{*}=(-1)^{n} D^{n}, p(x)=1$, las funciones $\emptyset_{i}(x)$ verifican que $\emptyset_{i}(x) \in P_{n} y \emptyset_{i}(x)$ tiene el coeficiente de la potencia de mayor grado igual a $(-1)^{n} / n !$. Imponiendo a estas funciones, para $1 \leq i \leq m-1$, que verifiquen (6) para $f(x)=x^{k-1}$, $k=1,2, \ldots m$, quedan definidos de manera unica los $\mathrm{m}-1$ polinomins $\emptyset_{i}(x), \quad 1 \leq i \leq m-1, y$ en consecuencia, mediante (2) y (3) una fórmula de cuadratura que es exacta para todo polinomio de grado menor o igual que $2 n-i$. 
Despues de imponer las condiciones anteriores se obtiene la expresión analitica de las $\emptyset_{i}$, que resulta ser:

$$
\left.\emptyset_{i}(x)=\sum_{k=0}^{\{n / 2\}}(-1)^{n+k} \frac{\Delta x_{j}}{2}\right)^{2 k} c_{k}\left(x-x_{i+\frac{1}{2}}\right)^{n-2 k}
$$

donde $x_{i+\frac{1}{2}}=\left(x_{i}+x_{i+1}\right) / 2, \Delta x_{i}=x_{i+1}-x_{i},[n / 2]$ sera igual a $(n-1) / 2$ si.n es impar y a $n / 2$ en el caso de ser $n$ par y

$$
\frac{(2 n-2 k) ! n !}{(n-2 k) ! k !(n-k) !(2 n) !}=c_{k}
$$

\section{PESO DE LAS FORMULAS}

Segun la expresión (2) y utilizando los operadores reducidos, asc como la expresión de las $p_{i}(x)$ dadas en (7), encontramos:

$$
\begin{aligned}
& A_{h i}=\sum_{k=0}^{[(h+1) / 2]}(-1)^{h+1+k_{2}-(h+1)} c_{h k}\left[(-1)^{h+1}\left(\Delta x_{i}\right)^{h+1}-\left\langle\Delta x_{i-1}\right)^{h+1}\right] \\
& 2 \leq i \leq m-1 ; 0 \leq h \leq n-1 \\
& A_{h i}=\sum_{\substack{k=0 \\
k=1}}^{[(h+1) / 2]}(-1)^{k} c_{h k}\left(\Delta x_{i}\right)^{h+1} 2^{-(h+1)} \\
& A_{h m}=\sum_{k=0}^{[(h+1) / 2]}(-1)^{h+k} c_{h k}\left(\Delta x_{m-1}\right)^{h+1} 2^{-(h+1)} \\
&
\end{aligned}
$$

viniendo en este caso la constante $c_{h k}$ dada por:

$$
c_{h k}=\frac{(2 n-2 k) ! n !}{(n+1-\hat{2} k) ! k !(n-k) !(2 n) !}
$$


Caso de nodos equidistantes.

En este caso las funciones $\emptyset_{i}(x), 1 \leq i \leq m-1$, se obtienen una a partir de la anterior por una simple traslación. Si llamamos $M=\Delta x_{i}$, a partir de las expresiones anterioxes de los pesos encontramos:

$A_{h i}=0 \quad 2 \leq i \leq m-1 ; h=1,3, \ldots, N^{*}$

$\begin{aligned} A_{h i}=A_{h m}=\frac{1}{2} A_{h i}= & \sum_{k=0}^{[(h+1) / 2]}(-1)^{k+h}(M / 2)^{h+1} C_{h k} \\ & h=0,2,4, \ldots, h^{*} ; \quad 2 \leq i \leq m-1\end{aligned}$

$A_{h i}=-A_{h m}=\sum_{k=0}^{[(h+1) / 2]}(-1)^{h+k+1}(M / 2)^{h+1} C_{h k}$

$$
\mathrm{h}=1,3, \ldots, \mathrm{N}^{\star}
$$

con $n^{*}=n-2$ si $n$ par ó $n-1$ si $n$ impar

$N^{*}=n-1$ si $n$ par $\delta n-2$ si $n$ impar.

Según los anteriores valores de los pesos, en este caso la expresión general. de la formula dada por (1) se convierte en $\left(M^{*}=(n-2) / 2\right.$ si $n$ par ó $(n-1) / 2$ si $n$ impar $):$

$$
\begin{aligned}
\int_{a}^{b} f(x) d x= & \sum_{h=0}^{M^{*}} A(2 h)\left[f^{2 h)}(a)+2 \sum_{i=2}^{m-1} f^{2 h)}\left(x_{i}\right)+f^{2 h)}(b)\right] \\
& \sum_{h=0}^{(h-2) / 2} A_{(2 h+1)}\left[f^{2 h+1)}(a)-f^{2 h+1)}(b)\right]+R
\end{aligned}
$$

$y$ en el caso de tomarse $m=2$, la formula se convierte en:

$$
\int_{a}^{b} f(x) d x=\sum_{h=0}^{n-1} A_{h}\left[f^{h)}(a)+(-1)^{h} f^{h)}(b)\right]+R(f)
$$

fórmulas exactas para polinomios de grados $2 n-1$.

ERROR.

El error se afronta a la vista de la expresion (3), teniendo en cuenta que para cualquier particion nos es conocida la expresión de $\emptyset_{i}(x)$. por otra parte $\emptyset_{i}$ posee sus $n$ ceros reales en 
en $\left(x_{i}, x_{i+1}\right)$ y están relacionados con los de los polinomios de Legenare. Todo lo anterior facilita la obtención de cotas de error, pudiendose dar la expresión analitica de las mismas en función de los ceros de los polinomios de Legenare, expresión que no se incluye por razón de espacio. 